\title{
O ensino a distância na formação em saúde: uma revisão integrativa de literatura
}

Vivian Breglia Rosa Vieira', Carla Rosane Paz Arruda Teo²

\begin{abstract}
Resumo
Pesquisas acerca da formação dos profissionais da área da saúde apontam a necessidade de mudanças substanciais, sobretudo no que se refere ao perfil profissional desejado e ao modelo pedagógico adotado. A necessidade se dá sobre a construção de uma aprendizagem mais construtivista, e, nessa perspectiva, a Educação a Distância (EaD) pode contribuir com este fim. O presente artigo teve por objetivo identificar como a literatura aborda a aplicação da educação a distância na formação de profissionais de saúde. Para isso, foi realizada uma revisão integrativa de literatura. Apesar de um considerável número de publicações terem sido listadas a partir da busca com os cruzamentos de termos elencados para o estudo (trinta e cinco), apenas nove atenderam aos critérios de inclusão definidos para esta revisão. Os estudos incluídos foram apresentados considerando-se seu objetivo, a metodologia empregada e os principais resultados encontrados. Conclui-se que a maioria das experiências publicizadas com a inclusão de EaD em saúde foi exitosa e conseguiu cumprir, mesmo que parcialmente, com suas metas.
\end{abstract}

\section{Palavras-chave}

Educação a Distância. Capacitação de Recursos Humanos em Saúde. Profissionais de Saúde.

1. Mestre em Ciências da Saúde pela Universidade Comunitária da Região de Chapecó, Santa Catarina, Brasil; professora da União das Faculdades dos Grandes Lagos, Brasil. E-mail: vbrv2801@hotmail.com.

2. Doutora em Ciência de Alimentos pela Universidade Estadual de Londrina, Paraná, Brasil; professora da Universidade Comunitária da Região de Chapecó, Santa Catarina, Brasil. E-mail: carlateo@unochapeco.edu.br. 


\title{
Distance education in health training: an integrative literature review
}

Vivian Breglia Rosa Vieira*, Carla Rosane Paz Arruda Teo**

\begin{abstract}
Research on the training of health professionals indicates the need for substantial changes, especially with regard to the desired professional profile and pedagogical model adopted. The need is to build a more constructivist learning, and in this perspective, Distance Education (EaD) can contribute to this end. The present article aimed to identify how the literature addresses the application of distance education in the training of health professionals. For this, an integrative literature review carried out. Although a considerable number of publications were listed from the search with the crossings of terms listed for the study (thirtyfive), only 9 met the inclusion criteria defined for this review. The included studies were presented considering their objective, the methodology used and the main results found. It was conclude that most of the experiences with the inclusion of $\mathrm{EaD}$ in health were successful and were able to meet, even partially, their goals.
\end{abstract}

\section{Keywords}

Distance Education. Health Human Resource Training. Health Professionals.

\footnotetext{
* Master in Health Sciences, Community University of the Region of Chapecó, State of Santa Catarina, Brazil; professor at the Union of Colleges of the Grandes Lagos. E-mail: vbrv2801@hotmail.com.

** PhD in Food Science, State University of Londrina, State of Paraná, Brazil; professor at the Community University of the Region of Chapecó, State of Santa Catarina, Brazil. E-mail: carlateo@unochapeco.edu.br.
} 


\section{Introdução}

Mudanças substanciais no processo de formação dos profissionais da área da saúde, sobretudo aquelas relacionadas aos métodos pedagógicos adotados, são necessárias para alinhar o perfil dos egressos com a demanda de saúde da população brasileira. A ênfase se dá na maior integração entre o mundo do ensino e o do trabalho, na formação generalista, no trabalho multiprofissional e interdisciplinar, na diversificação dos cenários de prática e na adoção de metodologias ativas de aprendizagem. Entretanto, segundo Santos et al. (2005), a tradução dessas demandas em conteúdos e atividades curriculares esbarra na rigidez dos modelos curriculares tradicionais. De maneira geral, os modelos curriculares ainda são fragmentados, divididos em ciclos básico e profissional, muitas vezes pouco integrados. Quanto ao enfoque pedagógico, frequentemente limita-se às metodologias tradicionais embasadas na transmissão do conhecimento, não privilegiando a formação crítica do acadêmico (BRASIL, 2007). De acordo com Brasil (2007), o processo de educação superior deve pressupor a utilização de metodologias de ensino e aprendizagem que possibilitem que os estudantes tomem seus lugares de sujeitos na construção do conhecimento. O professor, neste contexto, deve ser visto como facilitador e orientador do processo.

Considerando-se a velocidade com que se produzem conhecimentos e tecnologias no mundo atual, um dos objetivos fundamentais da aprendizagem no curso de graduação é o de aprender a aprender. Isso requer o desenvolvimento de habilidades na busca, seleção e avaliação crítica de dados e informações em livros, periódicos, bases de dados locais e remotas, além da utilização das fontes pessoais de informação, incluindo aquelas advindas de sua própria experiência. (BRASIL, 2007, p. 23).

A escolha da estratégia de ensino deve ser feita respeitando-se alguns critérios. Dentre eles podem ser destacados: o perfil dos estudantes, o tamanho da turma, o conteúdo da disciplina, a habilidade do professor em conduzir o processo, os recursos disponibilizados pela instituição de ensino, entre outros. De acordo com Mazzioni (2013), as estratégias de ensino utilizadas pelos professores devem ser capazes de motivar e de envolver os estudantes, deixando claro o papel que lhes cabe. O mesmo autor ainda destaca que

A habilidade do professor em identificar essas diferenças e escolher os processos de ensinagem que melhor se adapte as características dos alunos com os quais trabalha e que considere as características dos conteúdos em discussão, poderá fazê-lo mais bem-sucedido no seu ofício de educar. (MAZZIONI, 2013, p. 96).

É consenso, a necessidade de avanço para uma aprendizagem construtivista, e, nesse sentido, a Educação a Distância (EaD) pode contribuir para este fim. Esta modalidade de ensino deve ser considerada não apenas como uma alternativa, mas também como uma nova metodologia de ensinar e aprender, tendo em vista que não se restringe a transmissão de conhecimento (VARGAS et al., 2016). Vargas et al. (2016) afirmam que a proposta da EaD, em contextos atuais, se sustenta em um modo de ensinar e aprender globalizado, multidimensional, considerando a complexidade dos sujeitos e da sociedade. Segundo estes autores, no processo de ensinoaprendizagem em EaD, é necessário considerar aspectos biológicos, psíquicos, sociais, afetivos, históricos, econômicos, culturais e o modo 
como o sujeito está inserido na sociedade.

\section{A formação em saúde e as Diretrizes Curriculares Nacionais}

A discussão acerca da formação de profissionais de saúde torna-se importante como estratégia para superar o descompasso entre este processo e os princípios, diretrizes e necessidades do Sistema Único de Saúde (SUS) (BRASIL, 2006).

A formação do profissional de saúde vem passando por grandes contradições. Por um lado, a formação ainda segue o modelo médico-centrado, biologicista, preparando os profissionais para atuarem pontualmente a partir da queixa e da doença, em uma perspectiva individual, sem levar em conta as implicações do enfoque multiprofissional e da perspectiva interdisciplinar. De outro lado, é crescente a necessidade de um profissional preparado para atuar a partir de uma abordagem integral, com foco na Atenção Básica, na intersetorialidade e a partir de ações multiprofissionais, percebendo o usuário inserido em um contexto que é único e dinâmico.

No período de 2001 a 2004, o Conselho Nacional de Educação, por meio da Câmara de Educação Superior, publicou uma série de diretrizes específicas para os cursos de graduação da área da saúde, as Diretrizes Curriculares Nacionais (DCN) para a área da saúde (ALMEIDA, 2005). As DCN constituem um padrão geral de orientação para elaboração dos projetos pedagógicos e currículos das instituições de ensino superior (BRASIL, 2001). Essas diretrizes apontam a necessidade de incorporação do arcabouço teórico sobre o SUS nos projetos pedagógicos dos cursos, valorizam os postulados éticos, a cidadania, a epidemiologia e o processo saúde-doençacuidado, no sentido de garantir uma formação contemporânea de acordo com referenciais nacionais e internacionais de qualidade. Inovam, ainda, ao estimular a inserção precoce e progressiva do estudante no SUS, o que the garantirá conhecimento e compromisso com a realidade de saúde do seu país e sua região (BRASIL, 2001; INEP, 2006). Silveira (2004) destaca que as DCN são fruto de um processo histórico de questionamento da formação em saúde que envolveu, além dos órgãos competentes do Estado, diferentes segmentos da sociedade civil, constituindo um processo embasado nos princípios da legalidade e da legitimidade.

As DCN têm como ideia básica a flexibilização curricular, com vistas a possibilitar uma formação sólida que qualifique o graduando para o enfrentamento das mudanças do conhecimento e seus reflexos no mundo do trabalho (BRASIL, 2006). Soares e Aguiar (2010) citam que as DCN sugerem que o conteúdo principal não esteja mais centrado em matérias, duração e carga horária, mas sim no delineamento do perfil profissional, dos princípios que devem reger as profissões, bem comonas competênciase habilidades requeridas.

As diretrizes orientam para currículos que contemplem elementos de fundamentação essencial no seu campo do saber ou profissão, numa concepção de que o indivíduo deve aprender a aprender, engajado num processo de educação permanente. Propõe-se que os cursos de graduação sejam baseados em aprendizagem ativa, centrada no aluno, como sujeito da aprendizagem e no professor, como facilitador e mediador deste processo de ensino-aprendizagem. Enfocase o aprendizado baseado em competências, em evidências científicas, na solução de problemas e orientado para a comunidade. (BRASIL, 2006, p.13).

Ceccim e Feuerwerker (2004) referem que as DCN, mesmo reafirmando a posição de orientação ao sistema de saúde vigente, constituem-se apenas como uma recomendação, uma vez que, no Brasil, as universidades gozam de autonomia para fixação dos currículos 
de seus cursos. Os autores reforçam que novos passos, para além das diretrizes, são necessários para que as mudanças na formação ocorram efetivamente nas graduações em saúde (CECCIM; FEUERWERKER, 2004). Uma das estratégias que podem fortalecer a educação para o SUS, na formação de profissionais de saúde, é o aprimoramento do uso de tecnologias de educação, especialmente no contexto da EaD. A incorporação tecnológica na educação tem grande valor, pois proporciona conhecimento, atualização e aperfeiçoamento do profissional. Essa metodologia de ensino deve ser organizada de forma a trabalhar as necessidades educativas identificadas em cada grupo (GODOY; GUIMARÃES; ASSIS, 2014).

\section{Metodologia}

O estudo foi realizado por meio de uma revisão bibliográfica. Segundo Fogliatto (2007), a revisão bibliográfica é aquela que reúne ideias oriundas de diferentes fontes, visando construir uma nova teoria ou uma nova forma de apresentação para um assunto já conhecido. Seguiu-se, para tanto, os critérios de revisão integrativa de literatura.

A revisão integrativa foi desenvolvida conforme Ganong (1987), seguindo as etapas: formulação da pergunta de pesquisa; definição dos critérios de inclusão e exclusão dos estudos; seleção e tabulação dos estudos; análise e discussão dos resultados.

A realização deste estudo foi norteada pela seguinte questão: Como a literatura aborda a aplicação da educação a distância na formação de profissionais de saúde?

A busca das publicações científicas que permitissem responder à pergunta supracitada foi realizada utilizando-se os termos "educação a distância", "formação em saúde" e "profissionais de saúde". A coleta de dados foi realizada na base de dados Scielo, em agosto de 2016. Foram realizadas buscas avançadas, usando-se o cruzamento dos termos: "educação a distância" AND "formação em saúde" e "educação a distância" AND "profissionais de saúde". A seleção dos estudos ocorreu a partir dos seguintes momentos: busca do quantitativo de trabalhos apresentados na base; leitura individual dos títulos, resumos e trabalhos completos encontrados. Todos os estudos que atenderam aos critérios de inclusão e exclusão foram coletados e salvos em pastas específicas.

Os critérios de inclusão desse estudo foram: a) trabalhos publicados no formato de artigos científicos; b) trabalhos publicados no período: 2007 a 2016 (últimos 10 anos); c) trabalhos nos idiomas: inglês, português, espanhol; d) trabalhos disponíveis on-line na forma completa em acesso gratuito.

Já os critérios de exclusão compreenderam: a) artigos do tipo: revisões bibliográficas, cartas, resenhas, editoriais; b) publicações do tipo: livros; capítulos de livros; publicações governamentais; boletins informativos; teses; dissertações; monografias e trabalhos de conclusão de curso; d) estudos que não estão disponibilizados on-line no formato completo para análise; e) estudos duplicados.

Dos trabalhos incluídos foram extraídos os itens que alimentaram uma matriz construída para organização e análise dos dados. Na matriz foram elencados os seguintes itens: ano de publicação; título; referência completa; periódico; país; natureza da pesquisa; cenário de pesquisa; objetivos do estudo; principais aspectos dos resultados (focando no objetivo dessa revisão) e observações gerais. Após a organização dos dados, foi construído o presente artigo, abordando os principais aspectos dos trabalhos selecionados.

\section{Apresentação e análise dos dados}

Apesar de um considerável número de publicações terem sido listadas a partir da busca com os cruzamentos de termos 
(35), apenas nove atenderam aos critérios de inclusão definidos para esta revisão. A seguir, são apresentados os principais achados que emergiram da análise das nove publicações selecionadas conforme descrito anteriormente.

Godoy, Guimarães e Assis (2014) desenvolveram um estudo que teve por objetivo avaliar a educação permanente a distância para a equipe de enfermagem de Unidades Básicas de Saúde. Para isso, os autores realizaram um estudo descritivo, de abordagem qualitativa. Os sujeitos deste estudo foram 17 enfermeiros que apresentaram uma média de frequência acima de 50\% nas videoconferências do Projeto Telenfermagem da Escola de Enfermagem da Universidade Federal de Minas Gerais, no período de agosto a dezembro de 2011. A ferramenta elencada para a coleta de dados da pesquisa foi a videoconferência. $\mathrm{O}$ instrumento aplicado na videoconferência teve questões fundamentais, como: Qual a sua opinião em relação às atividades do Projeto Telenfermagem desenvolvidas no seu município?; Como você avalia a utilização das ferramentas de Telenfermagem no processo de capacitação da equipe e nas práticas assistenciais?; Descreva aspectos positivos e negativos quanto à utilização da videoconferência e teleconsultoria off-line e on-line.

De acordo com os autores, o estudo apresentou limitações no que diz respeito ao aceite dos enfermeiros para participar da pesquisa. Segundo eles, a videoconferência pode ter inibido a participação. Entretanto, apesar das dificuldades encontradas, os resultados apontaram que a educação a distância se configurou como uma estratégia de ensino-aprendizagem positiva para capacitar os enfermeiros em suas práticas assistenciais nas redes do SUS. Ainda se notou que a EaD permitiu a aproximação de profissionais com interesses comuns, já que a interligação e a conectividade global tornam possível o desenvolvimento de parcerias e o trabalho colaborativo, integrando instituições e abolindo distâncias. Os principais pontos negativos referidos pelos autores foram: a necessidade de atualizar os profissionais técnicos de informática dos municípios a fim de minimizar os problemas e melhorar a conectividade dependente das condições técnicas da rede, bem como a demanda em sensibilizar os teleconsultores em relação ao tempo desejável para dar o retorno, considerando as necessidades dos profissionais envolvidos em processos de tomada de decisão clínica (GODOY; GUIMARÃES; ASSIS, 2014).

Daza, Berretin-Felix e Machado (2014) buscaram verificar e caracterizar os requisitos necessários para viabilizar o emprego de um cyber tutor para orientação junto a Agentes Comunitários de Saúde (ACS) de oito equipes de Saúde da Família da Secretaria de Saúde do município de Monte Negro, Rondônia, que atendem à população urbana e rural. A pesquisa utilizou metodologia qualitativa, com fins exploratórios, por meio de interpretação dos discursos. Os dados quantitativos obtidos foram tabulados em planilha específica e submetidos à análise estatística descritiva. Após a realização de atividade de um curso (via EaD), foi realizada uma videoconferência com a finalidade de coletar os depoimentos dos ACS por meio de um roteiro de questões empregado pelos pesquisadores, procurando identificar o compromisso de todos os envolvidos na investigação e as dificuldades/facilidades/ sugestões/proposições a respeito do conteúdo do curso, assim como aos procedimentos de acesso e estudos na rede tecnológica (navegação) que o instrumento oferece.

Dos 51 ACS convidados, 49 aceitaram participar da pesquisa voluntariamente, e apenas oito $(15,69 \%)$ acessaram o conteúdo disponibilizado em EaD em tempo hábil. Os ACS manifestaram que, para fazer uso da ferramenta, não tiveram horários disponíveis durante a jornada de trabalho, por este motivo tiveram que estudar no horário de almoço ou 
após o expediente; três agentes $(37,5 \%)$ fizeram uso de LAN house, utilizando recurso financeiro próprio para ter acesso a um computador; dois (25\%) utilizaram o computador do seu local de estudo ou de outro emprego, dois (25\%) utilizaram uma única vez algum computador em casa de um familiar, e um (12,5\%) utilizou o computador próprio e fez o estudo em casa (DAZA; BERRETIN-FELIX; MACHADO, 2014).

Os resultados indicaram a conveniência de uso da EaD para educação continuada de trabalhadores da saúde, contudo, apontaram a necessidade de: implantação de uma política de educação permanente que inclua cursos a distância; conhecimentos básicos prévios de informática; conhecimentos básicos prévios a respeito da internet; acesso facilitado a computadores; tempo disponível para estudos, dentro e fora do horário de trabalho; incentivo financeiro para aquisição de computadores, ou estabelecimento de parcerias com empresas comerciais (LAN houses, cyber cafés, entre outros) que oportunizem os estudos; implantação de plano de carreira que incentive a progressão profissional (DAZA; BERRETIN-FELIX; MACHADO, 2014).

Oliveira et al. (2013) procuraram refletir acerca da importância da EaD oferecida pela Universidade Aberta do Sistema Único de Saúde (UNA-SUS) para a educação permanente dos profissionais de saúde do estado do Maranhão, principalmente para aqueles que se encontram distantes dos grandes centros urbanos. Para isso, desenvolveram um estudo de abordagem qualitativa. Os resultados apontaram que a educação a distância possibilitou aos profissionais o acesso à capacitação permanente. As ferramentas utilizadas constituíramse como meio de modificar a formação e execução de políticas na área da saúde.

Rangel et al. (2012) buscaram analisar a experiência do Curso de Especialização em Saúde Coletiva, com concentração em Gestão Pública Municipal, desenvolvido no estado da Bahia, visando qualificar gestores do SUS, mediante a política de Educação Permanente em Saúde (EPS).O curso teve carga horária de 360 horas, com realização de seis encontros presenciais obrigatórios e uso do ambiente virtual de aprendizagem Moodle da Universidade Federal da Bahia. O Moodle, por meio das estratégias pedagógicas propostas durante o curso, constituiu-se como um ambiente de interação direta entre os participantes, os professores e a equipe de coordenação, caracterizado por uma comunicação todos-todos. Participaram do estudo 166 profissionais. Destes, 39\% $(n=65)$ eram técnicos que atuavam na gestão municipal, $7 \%(n=12)$ profissionais que atuavam no SUS e $3 \% \quad(n=5)$ profissionais com experiência e/ou interesse na área de Saúde Coletiva. Nesse estudo, o principal desafio enfrentado na EaD foram as limitações de acesso à rede de computadores na região. Levantouse que, aproximadamente, $47 \%(n=77)$ dos cursistas acessaram a internet em suas próprias residências, $41 \% \quad(n=67)$ em seu local de trabalho, $7 \%(n=12)$ em lan-houses, 3\% $(n=5)$ em instituições de ensino e outros. Nesse sentido, os autores apontam que a escolha do fórum como principal ferramenta de comunicação do curso foi acertada por se tratar de um método assíncrono, permitindo aos cursistas entrarem no ambiente virtual de aprendizagem no momento em que conseguissem acesso à internet, participando das discussões, já que elas permaneciam registradas no ambiente. Foi verificado também, pelos autores, que alguns cursistas (os autores não citam quantos) abandonaram o curso por não se identificarem com a concepção de educação a distância adotada, que enfatizava a interatividade e a participação ativa. Aproximadamente, 16\% $(n=27)$ dos cursistas chegaram ao final do curso apresentando algum estranhamento com o modelo de participação, e, muitos destes, não conseguiram se sentir à vontade para realizar os estudos por meio do ambiente virtual. 
Entretanto, vários alunos superaram a fase do estranhamento, alcançando certa autonomia para ousar, criar, questionar, embora, às vezes, se limitassem apenas a responder o que era questionado (RANGEL et al., 2012).

Barilli, Ebecken e Cunha (2011) apresentaram uma pesquisa desenvolvida no Laboratório de Métodos Computacionais em Engenharia da Universidade Federal do Rio de Janeiro, em parceria com a Escola Nacional de Saúde Pública Sérgio Arouca da Fundação Oswaldo Cruz, abordando a integração da educação a distância e realidade virtual (RV) como resposta às demandas educativas da saúde pública que exigem o desenvolvimento de habilidades motoras. A ideia do estudo foi desenvolver um ambiente virtual de aprendizagem complementar, apoiado na tecnologia de realidade virtual, para contribuir com o desenvolvimento de habilidades ligadas aos procedimentos antropométricos (incluindose a calibração dos instrumentos, posicionamento dos pacientes, deslocamento de cursores, leitura e registro de resultados), para coleta de dados do Sistema de Vigilância Alimentar e Nutricional (SISVAN) do SUS. O curso criado destinava-se a profissionais e agentes locais de saúde, assim como para todo pessoal envolvido no SISVAN, possuía uma carga horária total de 180 horas e preconizava que o aluno deveria dedicar-se aos estudos por um tempo médio de quatro a seis horas semanais. O material didático do curso era composto por uma fita VHS, um CD-ROM, três livros e um ambiente virtual de aprendizagem restrito aos participantes e destinado à comunicação e interação.

O estudo apontou que 189 voluntários interagiram no ambiente virtual de aprendizagem. Alguns dos participantes interagiram em tempo real e outros de forma remota. Verificou-se que todos os participantes possuíam endereço eletrônico (e-mail) válido e que a maioria deles utilizava computador em casa e no trabalho, inclusive os ACS (sugerindo uma mudança importante no que concerne à democratização do acesso à informática). Cem por cento (100\%) dos participantes consideraram a navegação e orientação do ambiente virtual de aprendizagem claras. Os exercícios propostos foram considerados adequados também por todos os participantes. O total de participantes ainda declarou ter sentido prazer ao utilizar o sistema e que essa tecnologia colaborou com seu processo de aprendizagem. Nesse contexto, os autores apontaram a tecnologia de realidade virtual como um recurso pedagógico adequado para a formação profissional no campo da educação em saúde pública. Além disso, afirmaram que a realidade virtual pode colaborar com processos de formação profissional à distância que exijam desenvolvimento de habilidades motoras (BARILLI; EBECKEN; CUNHA, 2011).

Mezzari (2011) relatou a experiência de implementação de mudança de estratégia pedagógica na disciplina de Parasitologia e Micologia Médica, do curso de Medicina da Universidade Federal de Ciências da Saúde de Porto Alegre, incluindo neste contexto a educação à distância. A autora referiu que essa disciplina sempre foi oferecida na modalidade presencial e a mudança relatada consistiu em utilizar parte do ensino na modalidade a distância, com a aplicação do método de ensino da Aprendizagem Baseada em Problemas (ABP). Escolheu-se o Moodle como suporte para a elaboração deste modelo por favorecer a interação e a construção conjunta do conhecimento. $\mathrm{O}$ gerenciamento do ensino à distância, com uso do método ABP, foi realizado por uma tutora, aluna de mestrado do Programa de Pós-Graduação em Ciências da Saúde da UFCSPA e supervisionado pelos professores da referida disciplina. As ferramentas utilizadas, no Moodle, foram: link para um arquivo ou página; chat (ou bate-papo) e fórum de discussão. Além da avaliação do desempenho dos estudantes, foi realizada a avaliação da metodologia de ensino 
empregada. Os alunos, sem se identificarem, foram convidados a responder a um questionário sobre o uso da ferramenta Moodle. O objetivo era verificar a percepção deles sobre a utilização do método de ensino a distância em relação ao método presencial tradicional. Participaram do estudo 44 alunos, todos os matriculados na disciplina (MEZZARI, 2011).

De acordo com a autora, no primeiro chat participaram 14 alunos; no segundo, participaram 2 alunos; no terceiro, 8 alunos; e no quarto, e último chat, nenhum aluno participou. Este resultado mostrou uma baixa adesão dos estudantes à proposta, demostrando, talvez, a dificuldade de adaptação ou desinteresse pelo novo método. Apesar disso, quando questionados "você prefere o método tradicional de ensino, no qual o professor expõe o assunto e você estuda os conteúdos?", 40,91\% responderam não; $31,82 \%$ responderam que sim e $27,27 \%$ dos estudantes referiram preferir os dois métodos. A autora do estudo destaca, portanto, que o uso de metodologia mista (presencial e a distância) pode contribuir para o aprendizado de forma dinâmica, reflexiva e autônoma, complementado com uma avaliação contínua do aluno, seja de modo formal ou informal (MEZZARI, 2011).

Tomaz e Molen (2011) desenvolveram um estudo que teve por objetivo avaliar a aceitabilidade de um curso baseado em educação a distância entre os profissionais que atuam na Estratégia Saúde da Família. Para isso, foi realizado um inquérito transversal, com abordagem quantitativa. A população do estudo foi constituída por 115 médicos e 140 enfermeiros da Estratégia Saúde da Família do estado do Ceará. Um questionário autoaplicável foi criado especificamente para este estudo. A taxa de resposta dos participantes foi de 81,9\% (209 questionários devolvidos para 255 encaminhados).

Os resultados apontaram que a maioria dos participantes $(90,7 \% ; n=186)$, nunca teve uma experiência em $\mathrm{EaD}$. No que se refere ao acesso aos recursos de aprendizagem, os achados mostraram que uma boa proporção dos respondentes $(59,3 \% ; \mathrm{n}=124)$ tinha fácil acesso a computador; entretanto, uma considerável proporção $(40,7 \% ; n=85)$ não tinha. A maioria $(71,3 \% ; n=149)$ não tinha acesso fácil à internet. Apesar das dificuldades de acesso a computadores e internet, a maioria $(83,5 \% ; n=172)$ afirmou que gostaria de participar de um provável curso baseado em EaD e, neste contexto, certificou-se que esta pode ser uma estratégia adequada à educação permanente dos profissionais de Saúde da Família (TOMAZ; MOLEN, 2011).

Nunes, Franco e Silva (2010), por meio da educação a distância baseada em método fundamentado na teoria construtivista, buscaram promover cenários de interação, visando à construção de uma prática/pensamento integral em saúde. Foi utilizado, nesse estudo, o Teleduc, um ambiente de aprendizagem a distância desenvolvido por pesquisadores do Núcleo de Informática Aplicada à Educação da Unicamp. A análise dos dados baseouse nas interações dos alunos, na participação nos fóruns de discussão, nas edições dos exercícios individuais e dos portfólios de grupo. A pesquisa foi desenvolvida com base na resolução, pelos alunos, de "situaçõesproblema", individualmente e em atividades de grupo. Posteriormente, houve participação em fóruns de discussão criados pelos profissionais de saúde convidados. Participaram do estudo 6 alunos do sexto ano do curso de medicina, 9 profissionais da saúde das áreas de medicina de família, cardiologia, pediatria, cirurgia geral, patologia, assistência social, psicologia, nutrição e fisioterapia, e a pesquisadora, que exerceu papel de tutora. Os dados da pesquisa foram organizados quantitativa e qualitativamente.

Os dados qualitativos foram organizados 
em categorias: relações de interação, postura cooperativa, postura não cooperativa, geração de conhecimento técnico e geração de conhecimento integral. Os dados quantitativos apontaram 539 acessos ao ambiente, 696 acessos aos fóruns; 264 mensagens de diálogos (manifestações de trocas de ideias) e 6 mensagens de monólogo (simples exposição de ideias do interlocutor) nos fóruns. Constatou-se que o ambiente e o método foram capazes de promover encontros favoráveis, já que houve predomínio de diálogos. Verificou-se que os alunos da pesquisa demonstraram uma postura não cooperativa e que isso representou restrições nos processos de interação. Contudo, apesar das limitações ressaltadas, os alunos puderam interagir em um espaço multiprofissional/ interdisciplinar e demonstraram indícios de reflexões e mudança de postura necessária à cooperação e à prática integral em saúde. Os autores observaram a necessidade de emprego de elementos que otimizem as interações, conferindo qualidade pedagógica e proporcionando um contexto favorável, a fim de prolongar o aprendizado rumo a uma prática transformadora(NUNES; FRANCO;SILVA, 2010).

Jacomini, Piai e Figueiredo (2008) buscaram avaliar um curso de educação a distância sobre hepatite $\mathrm{C}$ dirigido a alunos de enfermagem. Este estudo realizou-se a partir do desenvolvimento de um curso ocorrido por meio de EaD, utilizando o ambiente virtual de aprendizagem Moodle. A duração do curso foi de cinco semanas. Em cada módulo era esclarecido o objetivo correspondente e oferecido um pequeno sumário. O conteúdo era disponibilizado aos estudantes em formato Word e PDF e a respectiva tarefa, por meio de questões discursivas e fórum de discussão. O término do curso se deu em uma aula presencial em que foi aplicada a avaliação final. Participaram do estudo 29 alunos, do 3 음 e $4^{\underline{0}}$ anos de graduação em enfermagem da
Universidade Federal de São Carlos. Desses, apenas 12 concluíram o curso. Os alunos que não participaram até o fim alegaram falta de tempo, por estarem em período de provas, estágios e trabalhos extracurriculares.

Este estudo mostrou que a EaD é uma estratégia viável para a realização de educação continuada para futuros profissionais de saúde, entretanto, o tempo de dedicação do aluno ao curso deve ser melhor dimensionado (JACOMINI; PIAI; FIGUEIREDO, 2008).

\section{Considerações finais}

A partir da pesquisa realizada para compor este trabalho, foi possível perceber que poucos escritos sobre a utilização da EaD em saúde têm sido publicados em revistas científicas. Isso parece evidenciar a baixa adesão ao emprego desse recurso como estratégia de ensino nos cursos de formação de profissionais de saúde ou, ainda, pode indicar falta de interesse em avaliar e/ou publicizar tais experiências. Neste contexto, fazse necessária a realização de maiores reflexões acerca da relação da EaD com a formação e qualificação de profissionais nessa área.

Sabe-se que, no campo da saúde, a EaD pode se fortalecer como uma possibilidade metodológica para otimização da aproximação da educação em saúde no Brasil com o sistema de saúde vigente. Entretanto, as discussões acerca do desenvolvimento de habilidades e competências frente ao uso da metodologia EaD ainda precisam ser ampliadas, especialmente aquelas que se relacionam com atividades práticas inerentes às profissões da saúde. Alguns desafios foram apontados, dentre elesosrelacionadoscomoacessodosindivíduosàs tecnologias que permitem aumentar a demanda pela metodologia de $\mathrm{EaD}$ e a continuidade dos participantes nas atividades propostas. Apesar disso, a maioria das experiências relatadas com a inclusão de EaD em saúde, apontadas neste 
estudo, mostraram-se exitosas e conseguiu cumprir suas metas, mesmo que parcialmente.

Cabe ressaltar ainda que as experiências mais exitosas são do campo da educação permanente em saúde, e as menos exitosas vieram da graduação. Este fato pode estar relacionado com a maturidade dos estudantes e/ou com a necessidade de um mínimo de formação prévia para que possam usufruir autonomamente das oportunidades em EaD.

\section{Referências}

ALMEIDA, M. J. Diretrizes Curriculares Nacionais para os cursos universitários da área da Saúde. 2. ed. Londrina: Rede UNIDA, 2005. 92 p.

BARILLI, E. C. V. C.; EBECKEN,N. F. F.; CUNHA, G. G. A tecnologia de realidade virtual como recurso para formação em saúde pública à distância: uma aplicação para a aprendizagem dos procedimentos antropométricos. Ciência \& Saúde Coletiva, Rio de Janeiro, v. 16, supl. 1, p. 1.2471.256, 2011.

BRASIL. Ministério da Saúde. A aderência dos cursos de graduação em Enfermagem, Medicina e Odontologia às Diretrizes Curriculares Nacionais. Brasília, DF: Ministério da Saúde, 2006.

. Ministério da Educação. Parecer CNE/CES no 1.133, de 7 de agosto de 2001. Diretrizes Curriculares Nacionais dos Cursos de Graduação em Enfermagem, Medicina e Nutrição. Brasília, DF: Ministério da Educação, 2001.

Ministério da Saúde. Ministério da Educação. Programa Nacional de Reorientação da Formação Profissional em Saúde - Pró-Saúde: objetivos e implementação e desenvolvimento potencial. Brasília: Ministério da Saúde, 2007.

CECCIM, R. B.; FEUERWERKER, L. C. M. Mudanças na graduação das profissões de saúde sob o eixo da integralidade. Caderno de Saúde Pública, Rio de Janeiro, v. 20, n. 5, p. 1.400-1.410, set./ out. 2004.

DAZA, M. P. M.; BERRETIN-FELIX,G.; MACHADO, M. A. M. P. Requisitos para utilização de cyber tutor com agentes comunitários de saúde. Revista CEFAC: Speech, Language, Hearing Sciences and Education Journal, São Paulo, v. 16, n. 2, p. 573-581, mar./abr. 2014.

FOGLIATTO, F. Organização de textos científicos. 2007. Disponível em: http://www.producao. ufrgs.br/arquivos/disciplinas/146_seminario_de_pesquisa_2_diretrizes_referencial_teorico.doc.

Acesso em: 22 set. 2016.

GANONG, L. H. Integrative reviews of nursing. Res Nurs Health, New York, v. 10, n. 1, p. 1-11, Feb. 1987. doi: https://doi.org/10.1002/nur.4770100103.

GODOY, S. C. B.; GUIMARÃES, E. M. P.; ASSIS, D. S. S. Avaliação da capacitação dos enfermeiros em unidades básicas de saúde por meio da telenfermagem. Esc. Anna Nery Revista de Enfermagem, Rio de Janeiro, v. 18, n. 1, p. 148-155, jan./mar. 2014.

INEP. Instituto Nacional de Estudos e Pesquisa. A trajetória dos cursos de graduação na saúde: 1991-2004. In: HADDAD, A. E. et al (Orgs.). v. 15. Brasília, DF: Inep/MEC, 2006. 
JACOMINI, R. A.; PIAI, T. H.; FIGUEIREDO, R. M. Avaliação de um curso de educação a distância sobre hepatite C. Investigación y Educación em Enfermería, Medelín, v. 26, n. 2, supl., p. 98-104, set. 2008.

MAZZIONI, S. As estratégias utilizadas no processo de ensino-aprendizagem: concepções de alunos e professores de ciências contábeis. Revista Eletrônica de Administração e Turismo - ReAT. v. 2, n. 1, p. 93-109, jan./jun. 2013.

MEZZARI, A. O uso da aprendizagem baseada em problemas (ABP) como reforço ao ensino presencial utilizando o ambiente de aprendizagem Moodle. Rev. Bras. Educ. Med. [online], Brasília, v. 35, n. 1, p. 114-121, 2011.

NUNES, T. W. N.; FRANCO, S. R. K.; SILVA, V. D. Como a educação a distância pode contribuir para uma prática integral em saúde? Rev. Bras. Educ. Med. [online], Brasília, v. 34, n. 4, p. 554564, 2010.

OLIVEIRA, A. E. F. et al. Educação a distância e formação continuada: em busca de progressos para a saúde. Rev. Bras. Educ. Med. [online], Brasília, v. 37, n. 4, p. 578-583, 2013.

RANGEL, M. L. et al. Redes de aprendizagem colaborativa: contribuição da educação a distância no processo de qualificação de gestores do Sistema Único de Saúde - SUS. Interface: Comunicação, Saúde, Educação [online], Botucatu, v. 16, n. 41, p. 545-556, abr./jun. 2012.

SANTOS, L. A. S. et al. Projeto pedagógico do programa de graduação em nutrição da Escola de Nutrição da Universidade Federal da Bahia: uma proposta em construção. Revista de Nutrição, Campinas, v. 18, n. 1, p. 105-117, jan./fev. 2005. doi: https://doi.org/10.1590/S141552732005000100010 .

SILVEIRA, J. L. G. C. Diretrizes Curriculares Nacionais para os cursos de graduação em Odontologia: historicidade, legalidade e legitimidade. Pesqui. bras. odontopediatria clín. integr, João Pessoa, v. 4, p. 151-156, maio/ago. 2004.

SOARES, N. T.; AGUIAR, A. C. Diretrizes curriculares nacionais para os cursos de nutrição: avanços, lacunas, ambiguidade e perspectivas. Revista de Nutrição, Campinas, v. 23, n. 5, p. 895-905, set./ out. 2010. doi: https://doi.org/10.1590/S1415-52732010000500019.

TOMAZ, J. B. C.; MOLEN, H. T. V. D. Compreendendo os profissionais de saúde da família como potenciais estudantes na educação à distância. Rev. Bras. Educ. Med. [online], Brasília, v. 35, n. 2, p. 201-208, 2011.

VARGAS, F. M. A. et al. A educação a distância na qualificação de profissionais para o Sistema Único de Saúde: meta estudo. Trab. Educ. Saúde, Rio de Janeiro, v. 14, n. 3, p. 849-870, set./dez. 2016.

Submetido em 27 de setembro de 2017.

Aprovado em 1 de março de 2018. 